
LUPUS (PIL)

\section{FRI0258 \\ COMPARISON OF ELISA AND MULTIPLEX TECHNIQUES FOR QUANTIFYING A URINE BIOMARKERS PANEL FOR LUPUS NEPHRITIS IN CHILDREN}

E.M. Smith ${ }^{1,2}$, M.W. Beresford ${ }^{1,2} .{ }^{1}$ Paediatric Rheumatology, Alder Hey Children's NHS Foundation Trust, ${ }^{2}$ Women and Children's Health, Institute of Translational Medicine, University of Liverpool, Liverpool, UK

Background: A urine 'biomarker panel' comprising alpha-1-acid-glycoprotein (AGP), ceruloplasmin (CP), transferrin (TF) and lipocalin-like-prostaglandin-D synthase (LPGDS) has been shown to cross-sectionally perform to an 'excellent' level for Lupus Nephritis (LN) identification in children.' Quantification of all four biomarkers by enzyme linked immunoabsorbant assay (ELISA) techniques is time consuming and costly. Therefore, novel methods of biomarker panel quantification are required to facilitate future urine biomarker led monitoring studies.

Objectives: The main objective was to compare the ability of ELISA and multiplex biomarker quantification techniques to differentiate active versus inactive LN when the biomarkers are considered individually and in combination.

Methods: The urinary biomarkers were quantified by both ELISA and a newly developed, custom multiplex platform in participants of the UK Juvenile Systemic Lupus Erythematosus (JSLE) Cohort Study. Multiplex assay development involved identification of appropriate antibody pairs, assessment of JSLE urine sample matrix effects and range finding in JSLE patient samples. Patients were categorised as having active $L N$ (renal domain BILAG score of $A, B$ and previous histological confirmation of LN) or inactive LN (renal BILAG score D or E). Firth's penalised binary regression with AUC ROC analysis was used to compare the ability of multiplex and ELISA assays to detect active LN disease state univariately and in combination.

Results: Biomarker analysis was undertaken on 54 JSLE patients (13 active, 41 in-active). Assessment of each biomarker univariately demonstrated similar AUC values regardless of the biomarker quantification technique; LPGDS (ELISA AUC 0.826, multiplex AUC 0.829), TF (ELISA AUC 0.829, multiplex AUC 0.996), CP (ELISA AUC 0.901, multiplex AUC 0.983), AGP (ELISA AUC 0.934, multiplex AUC 0.979) (see table 1). Combining the multiplex biomarker data in the same order as the original ELISA based study led to a similar progressive increase in AUC as biomarkers were added to the model (optimal model including AGP $+\mathrm{CP}$ + LPGDS+TF ELISA AUC $=0.951$, multiplex $=0.995$ ). For all biomarker combinations, the multiplex-derived AUC was higher than the ELISA AUC.

Abstract FRI0258 - Table 1. AUC values generated looking at each biomarker univariately

\begin{tabular}{lcc}
\hline Individual biomarkers & ELISA AUC & Multiplex AUC \\
\hline LPGDS & 0.826 & 0.829 \\
TF & 0.829 & 0.996 \\
CP & 0.901 & 0.983 \\
AGP & 0.934 & 0.979 \\
\hline
\end{tabular}

Abstract FRI0258 - Table 2. Comparison of biomarker combination AUC ROC values

\begin{tabular}{lcc}
\hline Biomarker combinations & ELISA AUC & Multiplex AUC \\
\hline AGP & 0.880 & 0.979 \\
AGP+CP & 0.937 & 0.986 \\
AGP+CP+LGPDS & 0.942 & 0.985 \\
AGP+CP+ LGPDS+TF & 0.951 & 0.995
\end{tabular}

Conclusions: This new LN urine biomarker panel multiplex assay has been shown to display a comparable ability for active $\mathrm{LN}$ disease state identification as compared to existing ELISA techniques. The major advantage to this approach is that it reduces cost, processing time and the volume of sample required, as compared to ELISA techniques, representing a key enabler for future clinical studies.

\section{REFERENCE:}

[1] Smith EM, Jorgensen AL, Midgley A, Oni L, Goilav B, Putterman C, Wahezi D, Rubinstein T, Ekdawy D, Corkhill R, et al. International validation of a urinary biomarker panel for identification of active lupus nephritis in children. Pediatr Nephrol 2017:32:283-295.

Disclosure of Interest: None declared DOI: 10.1136/annrheumdis-2018-eular.5187
B. Jacobs ${ }^{1}$, H. Leiss ${ }^{2}$, I. Gessl ${ }^{2}$, A. Puchner ${ }^{2}$, J. Smolen ${ }^{2}$, G. Stummvoll ${ }^{2}$. ${ }^{1}$ Department of Internal Medicine, Providence Saint Vincent Medical Center, Portland, USA; ${ }^{2}$ Department of Rheumatology, Medical University of Vienna, Vienna, Austria

Background: Pristane induced lupus (PIL) is an established murine model of induced systemic lupus erythematosus (SLE). Mice develop specific autoantibodies and show symptoms of SLE including arthritis, glomerulonephritis and haemorrhagic pulmonary capillaritis.

Objectives: To investigate the therapeutic effects of in vitro-induced regulatory $T$ cells (iTreg) in the murine model of PIL.

Methods: BALB/c mice were injected i.p. with $0.5 \mathrm{ml}$ of pristane (PIL) or PBS (control). Naive $\mathrm{CD}_{4}^{+}$thymocytes were sorted and cultured and cell suspensions with $>80 \% \mathrm{CD}^{+} \mathrm{FoxP}^{+}$cells (iTreg) were injected i.v. (i) once when PIL was induced $\left(5 \times 10^{6}\right.$ iTreg (iTreg-single) $)$ or (ii) every 4 weeks $\left(1 \times 10^{6}\right.$ iTreg, iTregrep). Mice were monitored for paw swelling and grip strength. After 8 months, histological analysis quantified cartilage degradation, number of osteoclasts, extent of inflammation and bone erosion. Glomerulonephritis and pneumonitis were quantified using the kidney biopsy score and a histomorphometric image analysis system; inflammatory tissue was analysed by tissue cytometry. Serum levels of auto-antibodies were measured by ELISA. Frequencies of B cells, activated and regulatory $\mathrm{CD}_{4}{ }^{+} \mathrm{T}$ cells and Th1, Th2, Th17 cells were measured by flow cytometry. FlowCytomix Pro was used to measure serum cytokines. RT-qPCR was used to measure expression levels of interferon (IFN)-signature and T-cell subset related as well as inflammation-associated genes.

Results: Monthly injections of $1 \times 10^{6}$ iTreg reduced the clinical and histological severity of PIL-arthritis. This was seen by a higher mean grip strength, less mean paw swelling, retardation of symptom onset and a lower summative arthritis severity score (figure A). There was significant reduction of arthritis severity in al histological parameters and in the percentage of affected mice with erosive arthritis (33\% of iTreg-rep mice vs $62 \%$ of PIL-mice). A single boost of $5 \times 10^{6}$ iTreg could not prevent joint manifestation, however a slight retardation in 'loss of grip strength' and significantly less erosive area was seen. In regards to cellular composition of the inflammatory tissue in paws, a significantly increased relative amount of CD4 +Foxp3+cells was seen in the iTreg-rep group compared to PIL group.

Repeatedly injected mice (iTreg-rep) had significantly less renal disease (glomerular activity score) (figure B) and pulmonary involvement (perivascular inflammatory area) (figure C) compared to PIL. iTreg-rep mice had significantly lower serum levels of disease-associated auto-antibodies (figure D). Upon restimulation, splenic $\mathrm{CD}^{+}$cells in iTreg-rep showed a less pronounced Th1, Th2 and Th17 response as well as lower percentages in B- and activated T-cells, whereas the percentage of Treg was higher than in all other groups (figure E). FlowCytomix analysis showed reduced cytokine production after repeated iTreg administration. Correspondingly, iTreg-rep showed decreased expressions of IFN-signature-, Tcell subset- and inflammatory cytokine related genes (figure F).

A

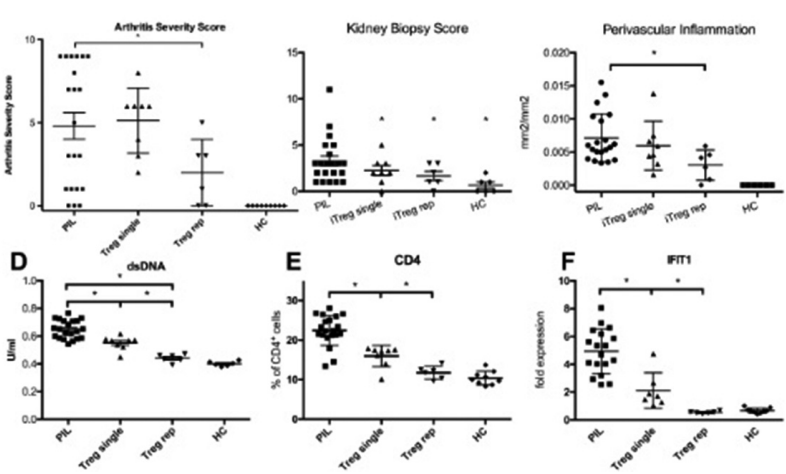

Conclusions: Repeated injections of iTreg ameliorate the clinical, histological, serological as well as the genetic severity of PIL-manifestations. A single boost of iTreg at time of disease induction does not prevent manifestations, but retards the 